\title{
Las sedes educativas del Plan FinEs2 como espacios de articulación de temporalidades
}

\author{
Un abordaje desde un estudio situado
}

\author{
FEDERICO MARTÍN GONZÁlEZ*
}

Este artículo analiza las experiencias educativas de estudiantes del Plan

Palabras clave

de Finalización de los Estudios Secundarios (FinEs2) a partir de una investigación doctoral realizada en dos barrios de clases populares de la ciudad de La Plata, Argentina, durante el periodo 2013-2017. Específicamente, nos preguntamos por cómo se configuran las denominadas sedes educativas del FinEs2 y cómo en dichos espacios se articulan temporalidades vinculadas a la política, al trabajo militante y a lo educativo. Para ello, utilizamos un corpus empírico construido a partir de distintas estrategias metodológicas: observaciones participantes y no participantes en distintos espacios barriales, entrevistas en profundidad a los distintos sujetos de esta política educativa y el abordaje de normativas del sistema educativo. Finalmente, concluimos que en la articulación de temporalidades se configura una serie de tácticas militantes y un modo de hacer escuela que logran poner en cuestión el carácter elitista y excluyente del nivel secundario en Argentina.

This article analyzes the educational experiences of some of the Secondary Studies Completion Plan (FinEs2) students, based on doctoral research carried out in two popular neighborhoods located in the city of La Plata, Argentina, during the period 2013-2017. We focused mainly on how FinEs2 educational venues are configured and how do they allow for the articulation of temporalities linked to politics, militant work and education. To do this, we used an empirical corpus adding different methodological strategies such as: participant and non-participant observations in different neighborhood spaces, indepth interviews with the different subjects of this educational policy and the approach to regulations of the educational system. Finally, we concluded that a series of militant techniques and a specific schooling tactics come together in the articulation of temporalities in such a way that brings into question the elitist and exclusive character of secondary level schools in Argentina.

Educación media

Desigualdad educativa

Temporalidad educativa

Participación política

Jóvenes-adultos

Keywords

Secondaryeducation

Educational inequality

Educational temporality

Political participation

Young adults

Recepción: 15 de junio de 2020 | Aceptación: 27 de mayo de 2021

DOI: https://doi.org/10.22201/iisue.24486167e.2022.175.59994

* Profesor de la cátedra Historia, política y gestión del sistema educativo argentino perteneciente al Departamento de Ciencias de la Educación de la Universidad Nacional de La Plata (UNLP) (Argentina). Becario posdoctoral del CONICET. Doctor en Ciencias Sociales. Líneas de investigación: desigualdades, educación y trabajo; políticas públicas de terminalidad educativa. Publicaciones recientes: (2019), "Volver a la escuela para alcanzar el buen trabajo. El ingreso a las fuerzas policiales y de seguridad como articulador de futuros laborales posibles en jóvenes varones estudiantes del Plan FinEs2 en Argentina”, Última Década, núm. 48, pp. 169-200; (2018, en coautoría con L. Crego), "La temporalidad escolar en cuestión. Negociaciones en una escuela secundaria y en un Plan FinEs", Propuesta Educativa, vol. 1, pp. 84-93. CE: federicomartin.gon@gmail.com 


\section{INTRODUCCIÓN}

En Argentina, la última Ley de Educación Nacional (LEN) 26.206, sancionada en el año 2006, fue un punto de quiebre en los ciclos de reforma educativa (Suasnabar, 2018). Esta normativa eliminó la organización planteada por las leyes de la década de los noventa, reestructuró el sistema educativo al reinstalar los niveles primarios y secundarios y provocó una serie de tensiones en la historia de la educación argentina. Específicamente, la obligatoriedad de la educación secundaria, implementada a partir de dicha ley, interrumpió la tradición excluyente del nivel configurada a partir de los primeros colegios nacionales a fines del siglo XIX (Tedesco, 1993).

Con base en el criterio elitista fundacional, los desafíos en torno a la efectiva universalidad de la educación secundaria constituyeron un punto de quiebre y actualizaron los interrogantes sobre educación y desigualdad. A partir de estos antecedentes, planteamos la idea de que la historia de la educación argentina se construye a partir de estudios y relatos sobre los ciclos de reforma que estructuran el sistema, pero también a partir del análisis de experiencias concretas que, enmarcadas en él, cuestionan o refuerzan características y tradiciones del mismo sistema.

Con el objetivo de aportar a los estudios sobre el nivel secundario en Argentina, este artículo analiza el proceso de configuración de las experiencias educativas enmarcadas en lo que la LEN nombró educación permanente de jóvenes y adultos. Para ello, retomamos una política pública de terminalidad educativa: el Plan de Finalización de los Estudios Secundarios (FinEs2), una política nacional gestionada por las provincias, que tiene como objetivo garantizar el derecho a la educación secundaria y su terminalidad a aquellos jóvenes y adultos que por distintos motivos no la han finalizado.

Para comprender con mayor profundidad la trayectoria del Plan FinEs2 es necesario enmarcarla en sus inicios, cuando en el año 2008 se delineó lo que posteriormente se presentó como un conjunto de políticas públicas de terminalidad educativa. En dicho año, el Plan FinEs (Plan nacional de finalización de estudios primarios y secundarios para jóvenes y adultos) pretendía garantizar espacios para aquéllos que, habiendo realizado el último año del nivel secundario, adeudaban materias para finalizar dicho trayecto. En una segunda instancia, se proyectaba desarrollar otra política de terminalidad destinada a quienes no habían iniciado o finalizado el nivel secundario. En línea con este último objetivo, en el año 2009 nació el Plan FinEs2, destinado a jóvenes y adultos mayores de 18 años y orientado a generar una experiencia de terminalidad completa de los estudios secundarios. En el caso de la Provincia de Buenos Aires se enmarcaron distintos planes y programas con objetivos similares (FinEs, Centros de Orientación y Apoyo, FinEs2, entre otros) dentro del Plan Provincial de Finalización de Estudios Obligatorios.

Con base en esta breve referencia histórica y del entramado institucional de la política educativa seleccionada, en este artículo nos preguntamos cómo se construyen las experiencias educativas en el Plan FinEs2. Retomamos la acepción filosófica de la categoría de experiencia en tanto se diferencia de la noción de práctica respecto de la posibilidad de interrumpir biografías y destinos sociales: para que una práctica se constituya como experiencia es necesario que varíe, que se modifique algo del orden de lo cotidiano, del mundo de lo vivido (Jay, 2009). De esta manera, estudiar experiencias de terminalidad educativa de jóvenes y adultos implica, en línea con Rockwell y Ezpeleta (1983), hacer foco en la cotidianeidad escolar e interpretar los múltiples procesos que allí acontecen. Específicamente, retomaremos esta perspectiva analítica para dar cuenta de cómo se configuraron las denominadas sedes educativas del FinEs2, cómo definieron sus tiempos y usos para hacer escuela los espacios barriales y cómo se articularon temporalidades vinculadas a la política, al trabajo militante y a lo educativo. 
Este artículo parte de una investigación doctoral que analizó las experiencias de jóvenes y adultos en el Plan FinEs2 en dos barrios de la ciudad de La Plata, capital de la provincia de Buenos Aires, Argentina, durante el periodo 2013-2017. El texto se encuentra estructurado en cuatro partes: en la primera se aborda el Plan FinEs2 como política pública de terminalidad educativa para luego ahondar en la perspectiva metodológica, la construcción del corpus empírico y el tipo de análisis utilizado en este artículo; en la segunda presentamos al nivel secundario desde un enfoque histórico para enmarcar lo que hemos nombrado como tensiones e interrupciones de la tradición excluyente; en la tercera nos enfocamos en el análisis de la experiencia educativa del Plan FinEs2, específicamente en las sedes educativas como espacios de articulación de temporalidades; $y$ finalmente recuperamos la noción de experiencia para establecer una serie de reflexiones generales.

\section{Sobre el Plan FinEs2 y la}

\section{PERSPECTIVA METODOLÓGICA DE LA INVESTIGACIÓN}

Para hacer explícita la perspectiva metodológica asumida es necesario plantear brevemente algunas referencias al diseño e implementación de la política analizada. La educación de adultos en Argentina presenta una historia de larga data con respecto a las articulaciones entre las esferas de gobierno estatal y distintos actores sociales y políticos en el desarrollo de experiencias educativas (Rodríguez, 1992). Luego de la sanción de la Ley de Educación Nacional (LEN) 26.206, en el año 2006, la denominada educación permanente de jóvenes y adultos presentó el objetivo de avanzar en la efectiva universalidad del secundario a jóvenes $\mathrm{y}$ adultos mayores de 18 años y recuperar dicha tradición histórica. De esta forma, se potenciaron los convenios entre los ministerios provinciales y los actores de la sociedad civil para la implementación de distintas políticas en territorios atravesados por complejas tramas de desigualdad social.

En un escenario de creación de nuevas institucionalidades y de jerarquización de la educación permanente de jóvenes y adultos como modalidad educativa, el Plan FinEs2 surgió en el año 2009 como una política pública de terminalidad educativa orientada a garantizar la obligatoriedad del nivel secundario a jóvenes y adultos que por distintos motivos no hubieran finalizado dicho trayecto educativo. A modo de organización de la descripción de esta política, explicaremos a continuación cuatro características de su implementación.

En primer lugar, el Plan FinEs2 proponía un formato alternativo para atender a la diversidad de trayectorias escolares en espacios educativos alejados de las formas tradicionales y gestionados cotidianamente por distintas organizaciones políticas y comunitarias. En el caso de la provincia de Buenos Aires, y a partir de un diagnóstico oficial del alcance insuficiente de las escuelas tradicionales de adultos, llamadas centros educativos del nivel secundario (CENS), se implementaron convenios entre las inspecciones de educación de adultos y distintas organizaciones que presentaban inserción territorial en barrios de sectores populares. Es así como, desde la narrativa de los funcionarios a cargo de la gestión se planteaba la idea de "descentralización" y la perspectiva de que la escuela tenía una segunda oportunidad al "ir al barrio".

En segundo lugar, la política de "descentralización” y convenios con distintas organizaciones permitió configurar sedes educativas del FinEs2 por fuera del dispositivo escolar clásico. De esta forma, los clubes, los locales de partidos políticos, las parroquias o iglesias y los centros municipales fueron los espacios en donde se desarrollaron las experiencias de terminalidad educativa.

En tercer lugar, quienes se desempeñaban como responsables de la gestión cotidiana del plan no estaban vinculadas a ámbitos educativos tradicionales, sino que se trataba de 
personas de las organizaciones reconocidas por esta política educativa como referentes de sede. Si bien las actividades que desarrollaban diariamente no eran remuneradas, los referentes se ocupaban de mantener los espacios abiertos, dialogar con los inspectores educativos a cargo de la coordinación de las sedes educativas de distintas regiones y administrar todos los registros de los docentes y estudiantes.

Por último, el formato del FinEs2 estaba enmarcado en una normativa que determinaba el carácter presencial, la asistencia a clase dos o tres veces por semana, la aprobación de cinco materias cuatrimestrales durante tres años y la posibilidad de establecer turnos de mañana, tarde y vespertino (nocturno). Sin embargo, el plan adquiría distintas particularidades debido a las tácticas cotidianas de los militantes y a las tradiciones de las organizaciones políticas que intervenían sobre lo normativo y sobre los espacios barriales en donde funcionaban las sedes educativas. En este sentido, la "descentralización" posibilitaba que se establecieran sedes educativas donde existía una demanda por parte de actores que conocían el barrio donde se encontraban insertos.

A partir de estas características de la política educativa, en este artículo se retoma una investigación que, desde un enfoque etnográfico, analizó las experiencias de terminalidad educativa de estudiantes jóvenes y adultos del Plan FinEs2 en la ciudad de La Plata durante el periodo 2013-2017. Las sedes educativas en donde se realizó el trabajo de campo son tres espacios reconocidos por su importante referencia territorial: un local perteneciente a un partido político, una parroquia y un Centro de Integración Barrial. Las tres sedes eran gestionadas por militantes de una organización política con presencia en la gestión de la municipalidad de la ciudad de La Plata hasta el año 2015.

Específicamente se analizará un corpus empírico construido a partir del diálogo entre distintas estrategias metodológicas. En primer lugar, observaciones participantes y no parti- cipantes en dos barrios de la zona oeste de la ciudad de La Plata, capital de la provincia de Buenos Aires; se registraron las construcciones cotidianas de los espacios de las sedes educativas del FinEs2 en donde es posible llevar a cabo el proceso de terminar la escuela secundaria. La posibilidad de sostener la presencia durante un tiempo prolongado permitió un descentramiento de las sedes educativas y la oportunidad de indagar la articulación de dimensiones políticas y educativas en otros espacios: las propias casas de los estudiantes y referentes de sede, movilizaciones y reuniones políticas y otros espacios públicos de los dos barrios. Es a partir de esto que se logró comprender lo educativo en su complejidad. En segundo lugar, entrevistas en profundidad a los distintos sujetos de esta política pública: estudiantes, referentes de sede y gestores en los niveles nacional, provincial y regional-municipal. En tercer lugar, el abordaje de normativas del sistema educativo y documentos de distintas organizaciones políticas vinculadas al desarrollo del Plan FinEs2 en los dos barrios estudiados.

Para este artículo dicho corpus empírico se procesó a través del software de análisis cualitativo de datos Atlas.ti. Para ello, construimos y utilizamos códigos analíticos enfocados a tres grandes niveles de análisis: trayectorias educativas, procesos educativos y articulaciones políticas. De esta forma, analizaremos distintas normativas oficiales, entrevistas en profundidad y escenas de campo que permitan dar cuenta de las articulaciones entre las dimensiones educativas y políticas en la configuración de experiencias de terminalidad educativa en el Plan FinEs2.

\section{El NiVEl SECUNDARio EN ARgENTINA. BREVES REFERENCIAS DESDE UNA CLAVE HISTÓRICA}

El Plan FinEs2 surgió en el año 2009 como parte de una política más amplia tendiente a garantizar la obligatoriedad del nivel secundario y enfrentar el carácter excluyente de 
la educación secundaria en Argentina. Para comprender este argumento, retomaremos a continuación algunas referencias sobre la historia del sistema educativo argentino.

La Ley 1.420 de Educación Común, Obligatoria y Gratuita, sancionada en el año 1884, constituyó la base fundante del sistema educativo argentino al configurar una estructura con un nivel primario obligatorio y un nivel secundario con una tradición selectiva y excluyente (Tedesco, 1993). De esta forma, la escuela primaria cumplió la compleja función de homogeneizar una sociedad fuertemente heterogénea y, por otra parte, se definió una enseñanza media de tipo preparatoria o propedéutica orientada especialmente a hombres que cumplirían funciones intelectuales y de dirigencia política en el naciente Estado (Giovine, 2001).

La tendencia hacia la masividad del proyecto de la escuela primaria común se enmarcó en lo que Puiggrós (1990) denomina el "sistema de instrucción pública centralizada estatal" (SIPCE), el cual refleja un elemento fundamental del espíritu de la época y de la generación de la década de los ochenta: la profunda confianza en la educación primaria para la creación de una narrativa en torno a los ideales de república, ciudadanía y modernidad (Puiggrós, 1990; Giovine, 2000). En oposición a la tradición que configuró el nivel primario, la secundaria nació con fines selectivos; en el año 1863 Bartolomé Mitre (presidente de la república de 1862 a 1868) estableció por decreto la creación de colegios nacionales en las capitales de las provincias y de esta forma dio inicio a la educación secundaria formal (Tedesco, 1993; Di Piero, 2016).

Lo expuesto nos permite sostener que el programa institucional que caracterizó a la escuela argentina cumpliría una doble función: por un lado, socializar a las generaciones en el marco de referencia de la cultura dominante y, por el otro, formar, especialmente en los niveles medios y superior, un hombre apto para cumplir roles en el sistema público:
Al fijar de esta manera su objetivo, la enseñanza se convirtió en patrimonio de una élite, porque el personal político que admite cualquier sistema, y más un sistema oligárquico como el de entonces, es necesariamente reducido. Esta característica - la de estar destinada a perpetuar en una élite las funciones directivas de la sociedad- es la que permite hablar de la educación argentina del siglo pasado como de una educación oligárquica (Tedesco, 1993: 63).

Si bien durante las décadas posteriores a su creación se da un proceso de ampliación de la matrícula del nivel secundario, los intentos de democratización continuaron con la estructura elitista que reflejaba el modelo de los colegios nacionales como espacios de privilegio y poder. Sin embargo, es posible identificar en la historia educativa argentina distintos momentos que buscaron combatir el carácter excluyente de este nivel. Uno de ellos está vinculado a las experiencias de las décadas de los cuarenta y cincuenta, específicamente en los dos primeros gobiernos peronistas, donde la ampliación del circuito de educación técnica en sus distintos niveles impulsó un proceso de democratización del sistema educativo (Dussel y Pineau, 1995). A pesar de que esta hipótesis no presenta total consenso al interior de la historiografía de la educación argentina (Plotkin, 1993; Somoza, 1997), la enseñanza técnica - como la creación de la Universidad Obrera Nacional en el año 1948, puesta en funcionamiento en 1952- constituyó una experiencia central en donde las nociones de trabajo, aprendiz y obrero se articularon en el discurso educativo de la época para formar parte de las controversias en torno a la definición del sujeto pedagógico (Puiggrós y Bernetti, 1993).

Pasadas las décadas de los setenta, ochenta y noventa, las normativas de los años 2000 -durante los gobiernos denominados kirchneristas (2003-2015) - constituyeron un segundo momento en tanto modificaron la estructura del sistema educativo e instalaron nuevos 
derechos y desafíos. Específicamente, la obligatoriedad de la educación secundaria y las políticas públicas de terminalidad educativa permitían la ampliación de la definición del sujeto pedagógico de dicho nivel. Antes de avanzar sobre este segundo momento recuperaremos algunos elementos para comprender las décadas anteriores y las dificultades para avanzar en la ampliación de los derechos educativos.

Braslavsky (1985), en una investigación post dictadura cívico-militar, retomó la idea de segmentación para dar cuenta de las lógicas de discriminación al interior del sistema educativo. La noción de segmento se constituyó como una categoría clásica en las investigaciones educativas que permitió comprender cómo en el sistema educativo conviven segmentos diferenciados según origen social. Una década más tarde, con la persistencia de estos fenómenos, Kessler (2002) y Tiramonti (2004) sostuvieron que la segmentación fue profundizada por los procesos de descentralización y fragmentación del sistema educativo llevados a cabo durante las reformas de la década de los noventa. Al respecto, Pineau (2013) sostiene que:

A diferencia de los segmentos, los fragmentos hacen referencia a espacios más o menos cerrados que congregan una serie de escuelas organizadas con un patrón cultural determinado. Si bien los fragmentos no son espacios homogéneos, tienen elementos culturales internos comunes... Normas, saberes y prácticas que responden a una matriz común que les dan unidad a la vez que los aíslan de los otros. Mientras que en la determinación de los segmentos primaban los criterios de distribución cuantitativa, en la fragmentación interviene la distribución cualitativa (Pineau, 2013: 41).

La consolidación del sistema educativo argentino a lo largo del siglo XX se dio a partir del desarrollo de una dinámica de inclusión y segmentación horizontal y vertical (Viñao, 2002). Desde esta perspectiva, es posible comprender a la escuela secundaria como producto de la combinación de dos tendencias: la continuidad del patrón institucional de los históricos colegios nacionales; y las lógicas de expulsión (Acosta, 2012). Retomando este marco conceptual, Acosta (2012) analiza la expansión de la matrícula durante el siglo XX y las dificultades estructurales para mejorar las tasas de egreso. En palabras de la autora:

...la masificación de la escuela media en la Argentina se produce desde una matriz de incorporación y expulsión en forma simultánea y estos procesos se relacionan con el modelo institucional de la escuela secundaria, cuyo origen radica en el modelo institucional del colegio nacional (Acosta, 2012: 2).

A lo largo del tiempo la tradición excluyente que caracterizó parte del sistema educativo en Argentina logró sedimentarse en prácticas institucionales y pedagógicas, por lo que la desigualdad y la meritocracia persistieron como criterio reinante (Di Piero, 2016). Es por ello que la sanción de la Ley de Educación Nacional 26.206 en el año 2006 y la obligatoriedad del nivel secundario constituyeron un punto nodal en la historia de la educación argentina. De hecho, en los análisis de la reforma educativa de los años noventa, la reestructuración del sistema educativo era considerada desde la siguiente perspectiva:

A pesar de que la reforma se dirige a los sectores más bajos de la población en el marco de una dinámica social descendente, es interesante hacer notar que hay una memoria histórica que le impide a los legisladores abrir el acceso de la escuela media (Tiramonti y Suasnabar, 2000: 146).

La idea de memoria histórica hace referencia a la persistencia del carácter elitista y excluyente del nivel secundario, propio del momento fundacional. Es en este sentido que concebimos a las normativas de los años 2000 como un segundo momento en el cual 
se produjeron ciertas tensiones en las lógicas selectivas que organizaron la enseñanza de dicho nivel. En este escenario, el Plan FinEs2 constituye una de las políticas públicas de terminalidad educativa enmarcadas en la modalidad que la última Ley de Educación Nacional (LEN) denominó como educación permanente de jóvenes y adultos.

Este segundo momento se caracteriza por la sanción del nuevo marco jurídico y la implementación de diversas políticas socio-educativas que, en su conjunto, constituyeron el telón de fondo de dos procesos diferentes pero complementarios: en primer lugar, la discusión sobre el formato de la escuela secundaria y la implementación de algunas variaciones de la forma escolar en la provincia de Buenos Aires; en segundo lugar, la puesta en marcha de un conjunto de políticas públicas que presentaron distintos objetivos, además de garantizar el acceso al nivel secundario (Finnegan y Brunetto, 2014; Nobile, 2016). Estos dos procesos renovaron interrogantes clásicos sobre las relaciones entre escuela, educación y desigualdad en un contexto de políticas educativas que, hasta el año 2015, se presentaban desde el paradigma de los derechos y de la posición activa del Estado nacional.

El desarrollo de estos debates y políticas se dio en diálogo con la definición de la desigualdad educativa como problema público. Tanto en la letra de la ley como en las resoluciones que acompañaron la norma, las relaciones entre desigualdad social y desigualdad educativa estuvieron presentes. De esta forma, se hace referencia a la importancia de atender a las desigualdades educativas de origen social, las características tradicionales del patrón organizacional de la escuela secundaria y, por ende, la necesidad de garantizar el cumplimiento de la obligatoriedad escolar.

A través de alternativas institucionales, pedagógicas y de promoción de derechos que se ajusten a los requerimientos locales y comunitarios, urbanos y rurales, mediante acciones que permitan alcanzar resultados de calidad equivalente en todo el país y en todas las situaciones sociales (LEN, 2006: 3).

Este breve recuento histórico nos permite comprender al sistema educativo argentino como una estructura cuyos niveles se encuentran enmarcados en tradiciones heterogéneas y contrapuestas. Específicamente, el carácter histórico de la educación secundaria permite comprender con mayor profundidad los desafíos ante la obligatoriedad de un nivel cuya historia se condensó en prácticas institucionales discriminatorias, pero también en disputas y experiencias democráticas. Es en este sentido que en esta sección recuperamos dos momentos en los que se puso en tensión la tradición excluyente, y cuyo origen data de finales del siglo XIX.

\section{UNA ORGANIZACIÓN QUE HACE ESCUELA.}

\section{TENSIONES Y TEMPORALIDADES EN LA experiencia del Plan FinEs2}

Las relaciones entre escuela secundaria y desigualdad social que hemos desarrollado en la sección anterior constituyen una de las dimensiones presentes en la investigación. Si bien los estudiantes y las militantes de la organización política que llevaban adelante las sedes del Plan FinEs2 reconocían la existencia de tres escuelas secundarias cercanas, también identificaban lógicas selectivas y de exclusión que dificultaban la terminalidad de los estudios. Para profundizar estos aspectos, presentaremos a continuación una escena en donde se establece un diálogo con un estudiante de la sede del FinEs2 de la parroquia.

Adrián había llegado temprano del trabajo en la panadería que el padre tiene en el barrio. Era la primera semana de julio del año 2016 y estaba a días de finalizar el trayecto formativo del FinEs2. Estaba contento de "terminar la escuela” y poder ingresar al Ejército Argentino, específicamente a la Infantería, donde trabajaba gran parte de su familia. 
Comenzamos a charlar y me contó que estaba contento por terminar "la escuela": "yo debería haber terminado hace mucho la escuela, pero dejé por la mala junta... hasta que me di cuenta que quería hacer algo y me puse las pilas...". Luego de varios intentos decidió inscribirse en la sede de la parroquia. Cuando iba "allá, a la 62 [en referencia a la escuela cercana al barrio] era distinto". Comenzamos a charlar sobre la escuela. Me explicó que algunas veces se quedaba "libre" porque faltaba mucho por trabajo o por problemas familiares. Frente a esa situación, lo que le molestaba era que cuando le preguntaban por qué, y contaba los motivos, le respondían que no importaba, porque su responsabilidad era ir. Adrián se preguntó: “ipara qué me preguntaban si siempre contestaban lo mismo?”. También hacía referencia a dimensiones pedagógicas para explicar la discontinuidad de su trayectoria. Así, comenzó a relatar su experiencia con la profesora de inglés que, cuando llegaba al aula, sólo anotaba en el pizarrón y no explicaba nada: "a la profesora no le importaba nada, acá [haciendo referencia al FinEs2 y sus docentes] te explican todas las veces que sean necesarias, se ponen con vos y te explican hasta que entendés". En relación a la contraposición que Adrián establecía entre el FinEs2 y su recorrido por la anterior escuela, el nombre de Carmen (la referente de sede educativa) y la relación con ella se hizo presente: "está bueno ir a algún lugar que sabés que a alguien le importás... ella siempre pregunta cómo estamos y cuando faltas ella se preocupa y habla con los profes" (diario de campo, 5/07/2016).

"Acá" y "allá" constituyen metáforas espaciales que Adrián retoma para analizar, en contraposición, su experiencia en la escuela y en las sedes del FinEs2. Esa reflexión le permitió identificar el lugar de la referente de sede y que en ese espacio él se sentía que importaba. Esta escena también permite comprender lo que anteriormente nombramos como la tradición excluyente del nivel secundario y su materialización en experiencias escolares concretas. Si bien la LEN, y específicamente la obligatoriedad de la educación secundaria, constituyó un punto de quiebre en el carácter selectivo del nivel secundario, no significó una transformación automática de las prácticas escolares cotidianas.

En este sentido, uno de los desafíos centrales de la ampliación de los derechos educativos radica en la posibilidad de superar las culturas institucionales y parte del universo simbólico docente. Prácticas escolares y discursos centrados en los pasados "tiempos dorados" de la escuela, posicionan a los nuevos sujetos en espacios que no se corresponden con las representaciones de los alumnos esperados (Freytes, 2012). Ahora bien, al interior del nivel secundario se han configurado experiencias que buscan combatir su carácter excluyente. De esta manera, a partir del estudio del funcionamiento cotidiano de las tres sedes del Plan FinEs2 abordaremos las discusiones sobre las disputas simbólicas en la definición y ampliación del sujeto pedagógico. Es decir, el reconocimiento de otro tipo de estudiantes, específicamente jóvenes y adultos que históricamente ha excluido la escuela, mediante mecanismos explícitos o implícitos, como sujetos estructurantes de los vínculos educativos gestados en las sedes del FinEs2 (Núñez, 2005).

\section{Tácticas militantes que hacen}

escuela en las sedes del Plan FinEs2

Problematizar el lugar de las organizaciones políticas en la configuración de experiencias educativas nos permite afirmar la importancia de las prácticas y luchas que los colectivos llevan a cabo cotidianamente con el objetivo de ampliar la definición y la cartografía de "lo posible" (Rancière, 2014). Rockwell y Ezpeleta (1983) plantean que, para analizar aquello que acontece en lo educativo dentro del horizonte del mundo de lo vivido, es necesario prestar atención a las tensiones entre el marco normativo y la experiencia escolar. Desde esta perspectiva, sostiene que para "hacer escuela" es necesario ofrecer otros mundos, otras lenguas $y$, de esta manera, la escuela hace Estado en un 
proceso de negociación y elaboración contingente. Por otra parte, Masschelein y Simons (2004) afirman que hacer escuela se encuentra asociado al ofrecimiento de un tiempo libre:

...una de sus características más radicales, pero que la definen esencialmente: que la escuela ofrece tiempo libre, que transforma conocimientos y destrezas en bienes comunes y, por lo tanto, que tiene el potencial para proporcionar a cada cual, independientemente de sus antecedentes, de su aptitud o de su talento natural, el tiempo y el espacio para abandonar su entorno conocido, para alzarse sobre sí mismo (Masschelein y Simons, 2004: 12).

Para estudiar la producción de otras formas de lo escolar en las sedes del FinEs2 pondremos el eje en el trabajo artesanal o, en términos de De Certeau (1996), en la "inventividad artesanal”. ¿Es posible pensar que en la gestión de las sedes del Plan FinEs2 y en el modo de hacer escuela se configura un hacer asociado a un estilo específico? Para abordar este interrogante retomamos el concepto de tácticas, en tanto nos permite enfocar las prácticas cotidianas y en las distintas maneras de hacer. A su vez, este concepto permite posicionar la agencia en un marco de distribución desigual de fuerzas y poderes, de lugares propios y no propios (De Certeau, 1996). En palabras del autor:

...la táctica depende del tiempo, atenta a "coger al vuelo" las posibilidades de provecho... Necesita constantemente jugar con los acontecimientos para hacer de ellos "ocasiones"... Lo hace en momentos oportunos en que combina elementos heterogéneos... Estas tácticas manifiestan también hasta qué punto la inteligencia es indisociable de los combates y los placeres cotidianos que articula... (De Certeau, 1996: L).

Las tácticas permiten dar cuenta de los movimientos que alteran la organización de lo espacial en un contexto marcado por "las cuotas desiguales de poder". La creatividad, entonces, habilita a pensar, trazar y delinear modos de hacer escuela ligados a la potencialidad de lo artesanal. En este sentido, las prácticas desplegadas por las referentes de sede para gestionar esta política pública de terminalidad educativa son resultado de articulaciones y convivencias de temporalidades asociadas a mundos que se encuentran vinculados. A partir de esta perspectiva analítica recuperaremos los siguientes interrogantes para abordar la articulación de las temporalidades de la política y de lo educativo: ¿cómo se crea un espacio educativo?, ¿cómo los espacios barriales configuran sus tiempos y usos para crear escuelas?

Como planteamos anteriormente, el trabajo de campo se realizó en tres sedes educativas: el "local" político, la parroquia y el CIB. Éstos forman parte del entramado territorial de la organización política y son habitados diariamente por militantes, entre ellas las referentes del Plan FinEs2, para el desarrollo de distintas actividades. Los relatos sobre las transformaciones de los espacios constituyen un elemento compartido en las narrativas de las militantes a cargo de las sedes educativas y reflejan la preocupación por configurar un lugar adecuado para el desarrollo del vínculo educativo.

Tanto el CIB y el "local", como la parroquia, fueron modificados al incorporar objetos característicos de la escuela como dispositivo (Dubet, 2004). Las mesas y sillas, los pupitres individuales con tapas fijas, los pizarrones y escritorios para los docentes delimitan las fronteras del aula al interior de los espacios, también utilizados para reuniones, talleres con niños y otras actividades. A pesar de la variedad de actividades que los espacios barriales condensan, a partir de las 17 horas predomina el protagonismo de las escenas que dan forma a las clases del FinEs2.

La preocupación por lo pedagógico y la resolución de lo necesario para el desarrollo del Plan FinEs2 dan cuenta de una de las dimensiones de la política en el trabajo de resignificación de los espacios. Analizar lo espacial 
contribuyó a estudiar la articulación de temporalidades en un mismo espacio y comprender que el despliegue de una táctica vinculada a la práctica militante forma parte de la creación y la gestión del espacio educativo. En este sentido, se podría afirmar que el FinEs2 implica un modo de hacer política asociado a la noción de espacio.

Para comprender el despliegue de la táctica de la gestión política de las sedes educativas presentamos una escena de campo que permitirá dar cuenta de cómo en un mismo lugar conviven y se articulan distintas temporalidades y narrativas. Transcribimos un fragmento del diario de campo del viernes 23 de octubre del 2015, dos días antes de las elecciones locales, provinciales y nacionales. ${ }^{1}$

Apenas pasadas las 17 horas se empezó a producir ese movimiento característico del local en las semanas previas a las elecciones nacionales: mientras ingresaban los estudiantes, salían y entraban grupos de mujeres que estaban realizando distintas actividades en el barrio, vinculadas, principalmente, a la campaña electoral. El local era el punto de encuentro donde se organizaban y dividían las actividades diarias a realizar... Cuando llegó la docente de la primera hora comenzó a hablar con la referente de la sede del CIB por un problema de asistencias de un estudiante. Al mismo tiempo, un grupo de mujeres pintaban unos carteles con los nombres de los candidatos electorales para luego colgar en la calle... $\mathrm{Al}$ comenzar la primera clase, el espacio en torno al escritorio donde las referentes del FinEs2 se sentaban adquirió otra dinámica: se hablaba en voz baja y las llamadas al celular eran atendidas en la vereda. Mientras la clase se desarrollaba, una de las referentes políticas del barrio me explicaba que para "hacer política” era necesario abordar problemáticas más importantes como "las mejoras en las casas del barrio” (diario de campo, 23/10/2015).
Esta escena permite ver cómo en un mismo espacio se articulan distintas temporalidades vinculadas, por un lado, a las clases del FinEs2 y, por el otro, al desarrollo de actividades de militancia. En este sentido, es necesario detenernos en lo que significa esta articulación, principalmente cuando el terreno de lo educativo suele estar ligado, desde las perspectivas tradicionales, a lo sagrado, a un espacio trascendental "fuera del mundo" y ajeno a la idea de lo militante (Dubet, 2004). Si bien las temporalidades de la política y lo educativo suelen ser consideradas como contradictorias entre sí, en estos espacios la convivencia se logra a partir de una preocupación política en torno a lo educativo. La temporalidad propia de la práctica militante permitió el desarrollo de esta política educativa desde una concepción donde el FinEs2 constituye una "oportunidad para el barrio" acorde a una nueva forma de "hacer política".

En relación con este aspecto, una de las docentes dijo que la preocupación por la educación y por otro tipo de problemáticas no se contradice con las prácticas que las militantes hacen diariamente:

...a mí me parece que hay un poco de todo... las encargadas y referentes de sedes tenían una preocupación porque había muchos pibes que no habían terminado el secundario en el barrio... y tenían claramente un compromiso con el barrio, no era que no lo tenían... eran mujeres que, si bien participaban desde la política y todo lo que tiene que ver con la movida de la política partidaria, no por eso ellas no tenían un compromiso (entrevista a docente, año 2017).

Con la idea de "la movida de la política partidaria" la docente hace referencia a la complementariedad entre la participación en un partido político, marchas, campañas electorales y el interés por las experiencias de los estudiantes en el FinEs2. En relación a lo

\footnotetext{
${ }^{1}$ En octubre del año 2015 se celebraron en Argentina elecciones locales (municipales), provinciales y nacionales para la elección de cargos correspondientes al poder ejecutivo y legislativo.
} 
que acontece en las sedes educativas o lo que hacen (o no hacen) las referentes de sede, muchos de los docentes sostienen un discurso de apoyo a las tácticas militantes llevadas a cabo para impulsar el FinEs2.

Sin embargo, la articulación de estas temporalidades en el trabajo militante provoca ciertas tensiones y demandas vinculadas a una percepción del Plan FinEs2 como "muy político"; es así que la preocupación por lo educativo promueve en las referentes de sedes y en las docentes el desarrollo de un trabajo de recreación de rituales y espacialidades escolares. Para abordar este aspecto, retomaremos el siguiente fragmento de entrevista a un estudiante:

R: si vamos a las materias era todo política... también con el tema de la sociedad y todo eso. No aprendimos mucho lo que era la escuelaescuela. Para mí era todo política.

P: ¿a quéllamas política?

R: Y que te... bueno... te hablaban mucho del gobierno, del pueblo... de todo eso, no me gustaba... me sacaba la cabeza. Había materias... o sea... sí tuvimos química, física, todo eso, pero por ejemplo nunca tuvimos biología, esas cosas nunca las tuvimos. Tuvimos otras materias... (entrevista a Darío, 21 años, estudiante, año 2017).

Parte de los estudiantes plantearon que el abordaje de determinados temas, como el feminismo y la problematización de los modelos sociales impulsados desde las políticas públicas, no constituían conocimientos que la escuela debería abordar. Si bien estos temas eran parte de los planes de estudio del nivel - establecidos por la resolución 6321/95 y la disposición 27/11 de la Dirección General de Cultura y Educación de la provincia de Buenos Aires- existía una demanda por una concepción de lo escolar relacionado con la trasmisión de saberes tradicionales "puros" o, en otros términos, ligados a una perspectiva de la escuela como un lugar vinculado al conocimiento legítimo (Crego, 2016). A esta crítica sobre los contenidos y formas de abordar las asignaturas se sumaba otra vinculada a la formalidad y a los rituales que tienen que ver con los dispositivos escolares tradicionales.

A partir de este tipo de cuestionamientos, algunos de los docentes dijeron que lo que intentaban instaurar en el FinEs2 estaba vinculado a una ruptura de una concepción tradicional de la escuela donde "el estudiante va sólo a estudiar y el conocimiento es visto como lo teórico". En palabras de una de las docentes:

Nuestras materias eran sobre política y era necesario problematizar esos temas. Mariano hacía rap y hablaba de un montón de estas cosas en sus letras. Juani hacía cumbia... y la cumbia también tiene que ver con esto... pero ninguno valoraba esos espacios o prácticas como lugares de saber y aprendizaje, ¿no? Yo creo que no quieren problematizar o hablar de estas cosas dentro de una escuela y exigen otras cosas: acá dame fórmulas, dame lo que sea, como esta visión mecánica de la escuela, vos venís, toma este texto, estudialo de memoria, mañana venís, me lo repetís y estás aprobado, ¿no? Me parece que molesta y genera tensiones a los profes que vamos más con una visión de educación popular, de deconstruir y reconocer a un otro, sus saberes, sus lógicas, pensando cómo abordar eso dentro de un aula, de la apropiación del espacio, de los vínculos, de la colectividad. Eso es lo que molesta, estar acostumbrado a algo que de repente genera resistencias... Creo que ese es nuestro desafío, estar ahí sabiendo que el sistema educativo propone otras lógicas, sabiendo que ellos están adaptados a esas lógicas que propone el sistema... y nosotros intentar romper con eso sin violentar demasiado (entrevista a docente, año 2017).

Frente a las demandas y tensiones planteadas por los estudiantes, las apuestas de los docentes por la interrupción de una lógica escolarizada o tradicional se hacen presentes en los espacios de las sedes educativas. En coincidencia con los planteos de Crego (2016), 
los docentes del Plan FinEs2 en la ciudad de La Plata presentan una preocupación pedagogía explícita por construir lógicas de trabajo "flexibles", atentas a las trayectorias de los estudiantes. El interés por problematizar las formas de enseñanza dialoga con el reconocimiento por parte de los docentes de lo que los estudiantes "van a buscar". Es así que las demandas por las prácticas y los rituales escolares hicieron eco en las distintas sedes del Plan FinEs2 y en las conversaciones pedagógicas entre los docentes y las referentes de sede. De esta manera se inició un proceso de configuración de un modo de hacer escuela que permitió la recreación de rituales escolares.

La cotidianeidad del FinEs2 se estructura a partir del trabajo en grupos, el uso del pizarrón, las exposiciones orales de los estudiantes, la organización de salidas educativas al barrio o a la Universidad Nacional de La Plata, la entrega periódica de trabajos prácticos, la firma de carpetas, los actos de egresados y la confección de boletines y certificados internos de acreditación de cursos en los que, cuatrimestre tras cuatrimestre, docentes y referentes asientan las calificaciones y el porcentaje de asistencia. Para profundizar el análisis de este aspecto relataremos a continuación una escena en la que es posible comprender las formas en que las tácticas cotidianas dan respuesta a las demandas de rituales escolares.

Estela, la referente de la sede de la parroquia, sacó orgullosa un folio de su cartera con impresiones. Le pregunté qué eran y me contó que con una de las docentes habían diseñado los nuevos boletines y se había encargado de imprimirlos en un papel grueso. Me pasó uno de los modelos para que los vea: los boletines estaban armados según la comisión y con las materias que correspondían según el cuatrimestre. Sólo era necesario completar los nombres, apellidos y documentos de cada estudiante. Otra de las referentes comenzó a gritar: "al fin tenemos boletines, los hicimos nosotras porque en Inspección de Adultos venían prometiéndonos, pero nunca los hicieron”. Los boletines tenían un recuadro donde cada fila correspondía a una materia, con celdas tanto para la nota numérica como para la firma del docente de la asignatura (diario de campo, 12/7/2016).

La iniciativa de la entrega de boletines y certificados para los egresados constituye una respuesta por parte de las referentes de sede, con ayuda de los profesores, respecto de las demandas de mayor institucionalización. Si bien esos documentos no son oficiales, con ellos se busca construir signos de legitimación a partir de la impresión de los logos de las distintas instituciones o niveles gubernamentales que forman parte de la estructura de gestión de dicha política educativa. Asimismo, son firmados por la inspectora de Educación de Adultos gracias a que las referentes de sede se ocupan de llevarlos a la Inspección con tiempo suficiente para tramitar la firma.

De alguna forma, estos objetos sintetizan los vínculos entre las demandas por la recreación de rituales y signos escolares y las tácticas militantes desplegadas por las referentes del FinEs2 para darles respuesta. A su vez, en las trayectorias educativas de jóvenes y adultos de clases populares la posesión de una certificación hace referencia a uno de los aspectos centrales del proceso de volver a la escuela: la legitimidad de un símbolo ligado a instituciones escolares tradicionales. De esta forma, la materialidad de las certificaciones se enmarca en trayectorias familiares donde el acceso a la escuela secundaria no había sido posible.

La creatividad por parte de los docentes y referentes para, en términos de De Certeau (1996), inventar distintos signos y rituales habilita a atribuir o añadir a las experiencias de terminalidad educativa en el Plan FinEs2 formalidades asociadas al imaginario de una escuela que los ha excluido. Las prácticas cotidianas de estos dos actores del FinEs2 posibilitan un modo de hacer escuela en las sedes educativas donde se recrean rituales y espacialidades ligadas a lo escolar. De esta 
manera, las articulaciones entre docentes y referentes, entre temporalidades de la política y de lo educativo, generan efectos virtuosos para responder a algunas de las críticas de los estudiantes en torno "a lo político" del FinEs2.

\section{Las sedes educativas como espacios de} articulación de temporalidades

El interrogante sobre cómo se configuran los tiempos y usos de los espacios nos permite ahondar en el análisis de la articulación de temporalidades vinculadas a la política, al trabajo militante y a lo educativo (Hall, 2010). Según Massey (2012), la producción de lo espacial no está dado y requiere el trabajo de la vinculación de dimensiones sociales que no se encuentran ligadas o unidas necesariamente.

Los lugares y los espacios, más que localizaciones con coherencia propia devienen focos de encuentro de lo no relacionado. Aún más, si eso es así, entonces lo espacial se convierte en generador de narrativas. Al poner en contacto distintas temporalidades, lo espacial provoca interacción, la cual pone en marcha nuevos procesos sociales (Massey, 2012: 139).

La convivencia de temporalidades heterogéneas en las sedes del FinEs2 refleja que la producción del espacio educativo no es algo que se encuentra dado a priori, sino que implica un trabajo sostenido y el despliegue de una serie de tácticas militantes asociadas a una forma particular de hacer escuela.

Las escenas de campo y los fragmentos de entrevistas recuperadas en las secciones anteriores nos permiten dar cuenta de que la articulación de temporalidades de la política y lo educativo se logra gracias a una preocupación sostenida por la resignificación de los espacios y por la interrupción de lógicas vinculadas a la desigualdad educativa. El desarrollo de estas tácticas refleja conjuntamente una forma de hacer escuela en las sedes del Plan FinEs2 que puede vincularse a la dimensión política de la temporalidad educativa y a las formas de verificación de los jóvenes y adultos como sujetos de derecho.

En el análisis de las convivencias y tensiones de estas temporalidades retomamos una concepción de la política alejada del economicismo y el moralismo (Quirós, 2011). La autora afirma que el clientelismo constituye un "acto de transgresión" al mezclar en la "política popular” lo que no debe ser mezclado: el dominio de la economía - asociado a la idea de satisfacción de necesidades y búsqueda de intereses personales- y el dominio de la política - vinculado al compromiso y a la entrega desinteresada por el bien común-(Quirós, 2011). A partir de estos aportes y del planteamiento de vínculos con el desarrollo de la política educativa, es posible afirmar que en el FinEs2 se combinan elementos que, desde una perspectiva tradicional, no deberían ser mezclados: la educación y el trabajo militante de las referentes de sede al interior de un partido político de la ciudad de La Plata. De esta forma, lo sagrado (es decir, lo educativo) y lo profano (la política) se combinan en los espacios de las sedes educativas (Dubet, 2004).

Esta perspectiva nos permitió enmarcar al FinEs2 en la trama política colectiva y reconocer que esta mezcla presenta algo virtuoso; es decir, el despliegue de distintas tácticas militantes permite el impulso y la gestión de esta política educativa y consolida un espacio donde los adultos y los jóvenes "tienen que estar adentro" a partir de un modo de hacer que verifica la ampliación del sujeto pedagógico. De la misma forma, posibilita que el "local", la parroquia y el CIB se configuren como espacios con presencia de símbolos de estatalidad. Las consultas constantes sobre los procesos de inscripción al Plan FinEs2 y el acceso a otro tipo de políticas sociales dan cuenta de la circulación de información entre los vecinos: allí es posible acceder a derechos.

Esta forma de configurar los espacios de las sedes del FinEs2 es producto de una combinación entre, por un lado, los efectos de la política pública que reconoce a los espacios barriales 
como escolares y, por otro lado, el despliegue de una serie de tácticas que contribuyen a configurar un modo de hacer escuela ligado a la idea de reconocimiento. Es a partir de este marco que es posible sostener que la articulación de temporalidades habilita la impresión de lógicas estatales y escolares en los espacios barriales y el sostenimiento de una temporalidad escolar que, en su conjunto, configura un modo específico de hacer escuela con efectos filiatorios (Redondo, 2018). Con tensiones, la ampliación del sujeto pedagógico y las distintas formas de trasmisión de conocimientos caracterizan el modo de hacer escuela.

\section{REFLEXIONES FINALES}

En este artículo nos preguntamos por las experiencias educativas en el Plan FinEs2, específicamente en torno a la configuración de los tiempos y usos de las sedes educativas. Retomando una perspectiva centrada en el análisis del mundo de lo vivido, planteamos que la articulación de temporalidades vinculadas a la política, al trabajo militante y a lo educativo posibilitó un modo de hacer escuela anclado en la verificación de los jóvenes y adultos como sujetos pedagógicos de un nivel educativo con una tradición fuertemente excluyente.

Para comprender la potencialidad de las experiencias de esta política educativa fue necesario enmarcar la pregunta desde una perspectiva histórica. Así, en la tercera sección abordamos breves referencias a la historia del sistema educativo argentino y planteamos dos momentos históricos que tensionaron el carácter elitista del nivel secundario. En este sentido, el estudio desde el enfoque de la experiencia nos permitió comprender que el campo educativo se configura a partir de estudios sobre ciclos de reformas, pero también del análisis de experiencias que buscan interrumpir y reordenar tradiciones fundacionales del mismo sistema (Grimberg, 2005). En línea con esta perspectiva, sostuvimos que la educación de adultos y las recientes políticas públicas de terminalidad educativa, como el Plan FinEs2, presentan un conjunto de características que modifican las definiciones del sujeto pedagógico.

A su vez, planteamos que la organización política que lleva adelante la gestión del Plan FinEs2 da respuestas a problemáticas que en otros escenarios o instituciones no se resolvían. Las tácticas militantes, que en este artículo hemos nombrado desde la perspectiva de De Certeau (1996) como creativas y artesanales, posibilitan el desarrollo cotidiano del Plan FinEs2 y la creación de espacios y experiencias afirmativas. Sostenemos, de esta manera, que estudiar experiencias enmarcadas en políticas educativas implica discutir las construcciones hegemónicas sobre determinadas definiciones que son estructurales del sistema educativo: qué se entiende por espacio escolar y por sujeto pedagógico. Retomando esta discusión, en el cuarto apartado desarrollamos la configuración de las sedes educativas como espacios en donde se articulan temporalidades asociadas a la política, al trabajo militante y a lo educativo. Si bien estas temporalidades suelen ser consideradas como contradictorias entre sí, en estos espacios la convivencia se logra a partir de una preocupación política en torno a lo educativo.

Finalmente, la perspectiva asumida nos permitió enmarcar al FinEs2 en la trama política colectiva y reconocer que esta articulación presenta efectos virtuosos. El despliegue de distintas tácticas vinculadas a la gestión, resignificación de las sedes educativas y recreación de rituales y signos escolares impulsó el desarrollo de una política pública que permitió la consolidación de un espacio que contribuye al cumplimiento del derecho a la educación secundaria. En este sentido, sostenemos que la dimensión política de la temporalidad educativa se refleja en la preocupación por la resignificación de los espacios barriales y la potencialidad del reparto de lo simbólico como una forma de interrumpir lógicas vinculadas a la desigualdad educativa. 
Acosta, Felicitas (2012), "La escuela secundaria argentina en perspectiva histórica y comparada: modelos institucionales y desgranamiento durante el siglo XX", Cadernos De História Da Educação, vol. 11, núm. 1, pp. 131-144.

Braslavsky, Cecilia (1985), La discriminación educativa, Buenos Aires, FLACSO/GEL.

Crego, María Laura (2016), "Resonancias de la variación escolar. Primer acercamiento a un plan de terminalidad educativa secundaria en la ciudad de La Plata”, Prisma Social. Revista de Ciencias Sociales, núm. 16, pp. 206-250.

De Certeau, Michel (1996), La invención de lo cotidiano. Artes, México, Universidad Iberoamericana.

Di Piero, María Emilia (2016), Justicia, meritocracia e igualitarismo. Procesos de producción y legitimación de desigualdades en escuelas secundarias universitarias en la Ciudad de La Plata, Tesis de Doctorado, Buenos Aires, FLACSO.

Dubet, Francois (2004), "Mutaciones institucionales y/o neoliberalismo", en Emilio Tenti Fanfani (comp.), Gobernabilidad de los sistemas educativos en América Latina, Buenos Aires, IIPE, pp. 15-44.

Dussel, Inés y Pablo Pineau (1995), "De cuando la clase obrera entró al paraíso: la educación técnica oficial durante el primer peronismo", en Adriana Puiggrós (dir.) y Sandra Carli (coord.), Discursos pedagógicos e imaginario social en el primer peronismo. Historia de la educación argentina, Buenos Aires, Editorial Galerna, pp. 107-176.

Finnegan, Florencia y Cecilia Brunetto (2014), “Acá hay un trabajo político: actores y relaciones en la producción del Plan FINES 2 Secundaria en la provincia de Buenos Aires”, La Plata, VIII Jornadas de Sociología de la UNLP, 3 al 5 de diciembre de 2014, en: http://www.memoria.fahce.unlp.edu.ar/trab_eventos/ev.4364/ ev.4364.pdf (consulta: 29 de mayo de 2020).

Freytes Frey, Ada (2012), "Las marcas de la segregación territorial en las escuelas secundarias básicas cercanas al río Reconquista, en José León Suárez (Gran Buenos Aires): discursos docentes y resistencias juveniles”, en Osvaldo Battistini y Gerad Mauger (comps), La difícil inserción de los jóvenes de clases populares en Argentina y Francia, Buenos Aires, Prometeo, pp. 121-152.

Giovine, Renata (2001), Culturas políticas, ciudadanías y gobierno escolar. Tensiones en torno a su definición: la provincia de Buenos Aires (1850-1880), Tesis de Maestría, Buenos Aires, FLACSO.

Grimberg, Mabel (2005), “En defensa de la salud y la vida'. Demandas e iniciativas de salud de agrupamientos sociales de la Ciudad de Buenos Aires y Conurbano Bonaerense -2001 a 2003-", Avá. Revista de Antropología, núm. 7, pp. 1-21.

Hall, Stuart (2010), "Sobre postmodernismo y articulación", en Eduardo Restrepo, Catherine Walsh y Víctor Vich (eds.), Sin garantías. Trayectorias y problemáticas en estudios culturales, Bogotá, Envión, pp. 75-94.

JaY, Martin (2009), Cantos de experiencia. Variaciones modernas sobre un tema universal, Barcelona, Paidós.

Kessler, Gabriel (2002), La experiencia escolar fragmentada. Estudiantes y docentes en la escuela media en Buenos Aires, Buenos Aires, IIPE-UNESCO.

Masschelein, Jan y Marteen Simons (2004), Defensa de la escuela. Una cuestión pública, Buenos Aires, Miño y Dávila.

Massey, Doreen (2012), Un sentido global del lugar, Barcelona, Icaria Editorial.

Nobile, Mariana (2016), "La escuela secundaria obligatoria en Argentina: desafíos pendientes para la integración de todos los jóvenes", Última Década, vol. 24, núm. 44, pp. 109-131.

NúÑEZ, Violeta (2005), "El vínculo educativo", en Hebe Tizio (coord.), Reinventar el vínculo educativo: aportaciones de la pedagogía social yel psicoanálisis, Barcelona, Gedisa, pp. 19-48.

Pineau, Pablo (2013), "Algunas ideas sobre el triunfo pasado, la crisis actual y las posibilidades futuras de la forma escolar", en Ricardo Baquero, Gabriela Diker y Graciela Frigerio (comps.), Las formas de lo escolar, Paraná, Editorial Fundación La Hendija, pp. 33-45.

Plotkin, Mariano (1993), Mañana es San Perón. Propaganda, rituales políticos y educación en el régimen peronista (1946-1955), Buenos Aires, Ariel.

Puiggrós, Adriana (1990), Sujetos, disciplina y curriculum en los orígenes del sistema educativo argentino (1885-1916), Buenos Aires, Galerna.

Puiggrós, Adriana y Jorge Luis Bernetti (1993), Peronismo: cultura política y educación (19451955), Buenos Aires, Galerna.

Quirós, Julieta (2011), "Moralismo y economicismo en la política popular: problemas de explicación socio-antropológica”, en Mabel Grimberg, Marcelo Ernandez Macedo y Virginia Manzano (comps), Antropología de las tramas políticas colectivas. Estudios en Argentina y Brasil, Buenos Aires, Antropofagia, pp. 235-256.

Rancière, Jacques (2014), El método de la igualdad. Conversaciones con Laurent Jeanpierre $y$ Dork Zabunyan, Buenos Aires, Claves.

Redondo, Patricia (2018), La escuela con los pies en el aire. Hacer escuela, entre la desigualdad y la emancipación, Río de Janeiro, UERJ. 
Rockwell, Elsie y Justa Ezpeleta (1983), "Escuela y clases subalternas", Cuadernos Políticos, núm. 37, pp. 70-80

Rodríguez, Lidia (1992), "La educación de adultos y la construcción de su especificidad", en Adriana Puiggrós (dir.), Escuela, democracia y orden (1916-1943), Buenos Aires, Galerna, pp. 255-295.

SomozA Rodríguez, Miguel (1997), “Interpretaciones acerca del proyecto educativo del primer peronismo. De agencia de adoctrinamiento a instancia procesadora de demandas", Revista Argentina de Historia de la Educación, núm. 1, pp. 163-184.

Suasnabar, Claudio (2018), "Los ciclos de reforma educativa en América Latina”, en Claudio Suasnabar, Laura Rovelli y Emilia di Piero (comps.), Análisis de política educativa. Teorías, enfoques y tendencias recientes en la Argentina, La Plata, Edulp, pp. 8-36.
Tedesco, Juan Carlos (1993), Educación y sociedad en la Argentina (1880-1945), Buenos Aires, Ediciones Solar.

Tiramonti, Guillermina (2004), “La fragmentación educativa y los cambios en los factores de estratificación", en Guillermina Tiramonti (comp.), La trama de la desigualdad educativa. Mutaciones recientes en la escuela media, Buenos Aires, Manantial, pp. 15-46.

Tiramonti, Guillermina y Claudio Suasnabar (2000), "La reforma educativa nacional en busca de una interpretación", Aportes para el Estado y la Administración Gubernamental, vol. 7, núm. 15, pp. 1-13.

VIÑaO, Antonio (2002), Sistemas educativos, culturas escolares y reformas: continuidades y cambios, Madrid, Morata. 